\title{
Multiple Mellin Convolution and I-function Transformation involving $r$ variables
}

\author{
Arif M. Khan \\ Head \& Asso. Professor, JIET Jodhpur, Raj. India - 342008 \\ Email: khanarif76@gmail.com
}

\begin{abstract}
An expression is obtained for an I-function of $r$ variables, transform of the Multiple Mellin convolution of the two functions in terms of the Multiple Mellin convolution of I-function transforms of the function involving $\mathrm{r}$ variables, it generalize the result given earlier by Kumbhat and Khan [5].
\end{abstract}

Keywords: Convolution, Multivariable I-Function, Multiple Mellin Convolution, Mellin Transformation.

\section{Introduction}

Kumbhat and Khan [5] have obtained an interesting relation involving a convolution of the Mellin type in connection with the I-function transformation. The aim of the present paper is to generalize their result to $r$ variables, I-function transformation.

Definition 1.1 : The multivariable I-function represented by Prasad [7] as

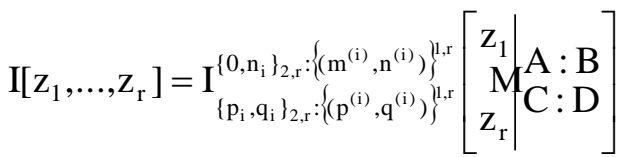

$$
\begin{aligned}
& =\frac{1}{(2 \pi \omega)^{r}} \int_{L_{1}} \ldots \int_{L_{r}} \psi\left(\zeta_{1}, \ldots, \zeta_{r}\right) \prod_{i=1}^{r}\left\{\phi_{i}\left(\zeta_{i}\right) z_{i}^{\zeta_{i}}\right\} d \zeta_{1} \ldots d \zeta_{r}
\end{aligned}
$$

where $\omega=\sqrt{-1}$,

$$
\psi\left(\zeta_{1}, \ldots \zeta_{\mathrm{r}}\right)=\frac{\prod_{\mathrm{k}=2}^{\mathrm{r}}\left[\prod_{\mathrm{j}=1}^{\mathrm{n}_{\mathrm{k}}} \Gamma\left(1-\mathrm{a}_{\mathrm{kj}}+\sum_{\mathrm{i}=1}^{\mathrm{k}} \alpha_{\mathrm{kj}}^{(\mathrm{i})} \zeta_{\mathrm{i}}\right)\right]}{\prod_{\mathrm{k}=2}^{\mathrm{r}}\left[\prod_{\mathrm{j}=\mathrm{n}_{\mathrm{k}}+1}^{\mathrm{p}_{\mathrm{k}}} \Gamma\left(\mathrm{a}_{\mathrm{kj}}-\sum_{\mathrm{i}=1}^{\mathrm{k}} \alpha_{\mathrm{kj}}^{(\mathrm{i})} \zeta_{\mathrm{i}}\right)\right]}
$$




$$
\begin{gathered}
\times \frac{1}{\prod_{\mathrm{k}=2}^{\mathrm{r}}\left[\prod_{\mathrm{j}=1}^{\mathrm{q}_{\mathrm{k}}} \Gamma\left(1-\mathrm{b}_{\mathrm{kj}}+\sum_{\mathrm{i}=1}^{\mathrm{k}} \beta_{\mathrm{kj}}^{(\mathrm{i})} \zeta_{\mathrm{i}}\right)\right]} \\
\phi_{\mathrm{i}}\left(\zeta_{\mathrm{i}}\right)=\frac{\left[\prod_{\mathrm{k}=1}^{\mathrm{m}^{(\mathrm{i})}} \Gamma\left(\mathrm{b}_{\mathrm{k}}^{(\mathrm{i})}-\beta_{\mathrm{k}}^{(\mathrm{i})} \zeta_{\mathrm{i}}\right)\right]\left[\prod_{\mathrm{j}=1}^{\mathrm{n}^{(\mathrm{i})}} \Gamma\left(1-\mathrm{a}_{\mathrm{j}}^{(\mathrm{i})}+\alpha_{\mathrm{j}}^{(\mathrm{i})} \zeta_{\mathrm{i}}\right)\right]}{\left[\prod_{\mathrm{j}=\mathrm{n}^{(\mathrm{i})}+1}^{\mathrm{p}^{(\mathrm{i})}} \Gamma\left(\mathrm{a}_{\mathrm{j}}^{(\mathrm{i})}-\alpha_{\mathrm{j}}^{(\mathrm{i})} \zeta_{\mathrm{i}}\right)\right]\left[\prod_{\mathrm{k}=\mathrm{m}^{(\mathrm{i})}+1}^{\mathrm{q}^{(i)}} \Gamma\left(1-\mathrm{b}_{\mathrm{k}}^{(\mathrm{i})}+\beta_{\mathrm{k}}^{(\mathrm{i})} \zeta_{\mathrm{i}}\right)\right]}
\end{gathered}
$$

$\forall \mathrm{i} \in\{1, \ldots, \mathrm{r}\}$. Also,

$$
\begin{array}{ll}
\left\{0, \mathrm{n}_{\mathrm{i}}\right\}_{2, \mathrm{r}} & :=0, \mathrm{n}_{2}: \ldots: 0, \mathrm{n}_{\mathrm{r}}, \\
\left\{\mathrm{p}_{\mathrm{i}}, \mathrm{q}_{\mathrm{i}}\right\}_{2, \mathrm{r}} & :=\mathrm{p}_{2}, \mathrm{q}_{2}: \ldots: \mathrm{p}_{\mathrm{r}}, \mathrm{q}_{\mathrm{r}}, \\
\left\{\left(\mathrm{m}^{(\mathrm{i})}, \mathrm{n}^{(\mathrm{i})}\right)\right\}^{1, \mathrm{r}} & :=\left(\mathrm{m}^{(1)}, \mathrm{n}^{(1)}\right) \ldots ;\left(\mathrm{m}^{(\mathrm{r})}, \mathrm{n}^{(\mathrm{r})}\right), \\
\left\{\left(\mathrm{p}^{(\mathrm{i})}, \mathrm{q}^{(\mathrm{i})}\right)\right\}^{1, \mathrm{r}} & :=\left(\mathrm{p}^{(1)}, \mathrm{q}^{(1)}\right), \ldots ;\left(\mathrm{p}^{(\mathrm{r})}, \mathrm{q}^{(\mathrm{r})}\right),
\end{array}
$$

$$
\begin{aligned}
& \left.\mathrm{A}:=:\left\{\left(\mathrm{a}_{\mathrm{ij}} ; \alpha_{\mathrm{ij}}^{(1)}, \ldots, \alpha_{\mathrm{ij}}^{(\mathrm{i})}\right)_{1, \mathrm{p}_{\mathrm{i}}}^{2, \mathrm{r}}\right\} \quad:=\left(\mathrm{a}_{2 \mathrm{j}} ; \alpha_{2 \mathrm{j}}^{(1)}, \alpha_{2 \mathrm{j}}^{(2)}\right)_{1, \mathrm{p}_{2}} ; \ldots ;\left(\mathrm{a}_{\mathrm{rj}} ; \alpha_{\mathrm{rj}}^{(1)}, \alpha_{\mathrm{rj}}^{(\mathrm{r})}\right)_{1, \mathrm{p}_{\mathrm{r}}}\right) \\
& \mathrm{B}:=:\left\{\left(a_{\mathrm{j}}^{(\mathrm{i})} ; \alpha_{\mathrm{j}}^{(\mathrm{i})}\right)_{1, \mathrm{p}^{(\mathrm{i})}}^{1, \mathrm{r}}\right\} \quad:=\left(\mathrm{a}_{\mathrm{j}}^{(1)}, \alpha_{\mathrm{j}}^{(1)}\right)_{1, \mathrm{p}^{(1)}} ; \ldots ;\left(\mathrm{a}_{\mathrm{j}}^{(\mathrm{r})}, \alpha_{\mathrm{j}}^{(\mathrm{r})}\right)_{1, \mathrm{p}^{(\mathrm{r})}} \\
& \left.\mathrm{C}:=:\left\{\left(\mathrm{b}_{\mathrm{ij}} ; \beta_{\mathrm{ij}}^{(1)}, \ldots, \beta_{\mathrm{ij}}^{(\mathrm{i})}\right)_{1, \mathrm{q}_{\mathrm{i}}}^{2, \mathrm{r}}\right\} \quad:=\left(\mathrm{b}_{2 \mathrm{j}} ; \beta_{2 \mathrm{j}}^{(1)}, \beta_{2 \mathrm{j}}^{(2)}\right)_{1, \mathrm{q}_{2}} ; \ldots ;\left(\mathrm{b}_{\mathrm{rj}} ; \beta_{\mathrm{rj}}^{(1)}, \beta_{\mathrm{rj}}^{(\mathrm{r})}\right)_{1, \mathrm{q}_{\mathrm{r}}}\right\} \\
& \mathrm{D}:=:\left\{\left(\mathrm{b}_{\mathrm{j}}^{(\mathrm{i})} ; \beta_{\mathrm{j}}^{(\mathrm{i})}\right)_{1, \mathrm{q}^{(\mathrm{i})}}^{1, \mathrm{r}}\right\} \\
& :=\left(\mathrm{b}_{\mathrm{j}}^{(1)}, \beta_{\mathrm{j}}^{(1)}\right)_{1, \mathrm{q}^{(1)}} ; \ldots ;\left(\mathrm{b}_{\mathrm{j}}^{(\mathrm{r})}, \beta_{\mathrm{j}}^{(\mathrm{r})}\right)_{1, \mathrm{q}^{(\mathrm{r})}}
\end{aligned}
$$

such that $n_{i}, p_{i}, q_{i}, m^{(i)}, n^{(i)}, p^{(i)} q^{(i)}$ are non-negative integers and all $a_{i j}, b_{i j}, \alpha_{i j}, \beta_{i j}$, $a_{j}^{(i)}, b_{j}^{(i)}, \alpha_{j}^{(i)}, \beta_{j}^{(i)}$ are complex numbers and the empty product denotes unity.

The contour integral (1.1) converges, if

$$
\left|\arg \mathrm{Z}_{\mathrm{i}}\right|<\frac{1}{2} \mathrm{U}_{\mathrm{i}} \pi, \mathrm{U}_{\mathrm{i}}>0, \mathrm{i}=1, \ldots, \mathrm{r}
$$

where

$$
\begin{array}{r}
U_{i}=\sum_{j=1}^{n^{(i)}} \alpha_{j}^{(i)}-\sum_{j=n^{(i)}+1}^{p^{(i)}} \alpha_{j}^{(i)}+\sum_{j=1}^{m^{(i)}} \beta_{j}^{(i)}-\sum_{j=m^{(i)}+1}^{q^{(i)}} \beta_{j}^{(i)}+\left(\sum_{j=1}^{n_{2}} \alpha_{2 j}^{(i)}-\sum_{j=n_{2}+1}^{p_{2}} \alpha_{2 j}^{(i)}\right) \\
+\ldots+\left(\sum_{j=1}^{n_{r}} \alpha_{r j}^{(i)}-\sum_{j=n_{r}+1}^{p_{r}} \alpha_{r j}^{(i)}\right)-\left(\sum_{j=1}^{q_{2}} \beta_{2 j}^{(i)}+\ldots+\sum_{j=1}^{q_{r}} \beta_{r j}^{(i)}\right)
\end{array}
$$

and $\mathrm{I}\left[\mathrm{z}_{1}, \ldots, \mathrm{z}_{\mathrm{r}}\right]=\mathrm{O}\left(\left|\mathrm{z}_{1}\right|^{\alpha_{1}}, \ldots,\left|\mathrm{z}_{\mathrm{r}}\right|^{\alpha_{\mathrm{r}}}\right), \max \left\{\left|\mathrm{z}_{1}\right|, \ldots,\left|\mathrm{z}_{\mathrm{r}}\right|\right\} \rightarrow 0$, where $\alpha_{\mathrm{i}}=\min _{1 \leq \mathrm{j} \leq \mathrm{m}^{(\mathrm{i})}} \mathfrak{R}\left(\mathrm{b}_{\mathrm{j}}^{(\mathrm{i})} / \beta_{\mathrm{j}}^{(\mathrm{i})}\right)$, and $\beta_{\mathrm{i}}=\max _{1 \leq \mathrm{j} \leq \mathrm{n}^{(\mathrm{i})}} \mathfrak{R}\left(\mathrm{a}_{\mathrm{j}}^{(\mathrm{i})}-1 / \alpha_{\mathrm{j}}^{(\mathrm{i})}\right), \mathrm{i}=1, \ldots, \mathrm{r}$. For the condition of convergence and analyticity of multivariable I-function we refer $[7,8]$ 
Definition 1.2 : Generalized Mellin convolution can be taken in the form, given by Datta \& Arora [1]

$$
(\mathrm{k} * \mathrm{f})\left(\mathrm{y}_{1}, \mathrm{y}_{2}, \ldots . . \mathrm{y}_{\mathrm{r}}\right)=\int_{0}^{\infty} \ldots \int_{0}^{\infty} \prod_{\mathrm{i}=1}^{\mathrm{r}} \mathrm{x}_{\mathrm{i}}^{-1} \mathrm{k}\left(\frac{\mathrm{y}_{1}}{\mathrm{x}_{1}}, \ldots \frac{\mathrm{y}_{\mathrm{r}}}{\mathrm{x}_{\mathrm{r}}}\right) \mathrm{f}\left(\mathrm{x}_{1}, \ldots \mathrm{x}_{\mathrm{r}}\right) \cdot \mathrm{dx}_{1}, \mathrm{dx}_{2} \ldots \mathrm{dx} \mathrm{x}_{\mathrm{r}}
$$

Further the Mellin convolution of two I functions involving r-variables given by Datta \& Arora [1] as

Theorem : Suppose the condition (9) to be satisfied and $\mu_{\mathrm{i}}>0, \mathrm{v}_{\mathrm{i}}>0, \mathrm{n}_{\mathrm{i}}=0, \mathrm{n}_{\mathrm{i}}^{\prime}=0, \mathrm{i} \in\{1, \ldots, \mathrm{r}\}$. Then the Multiple Mellin transform of the product of two I-function of $\mathrm{r}$ variable.

$$
\begin{aligned}
& (\Re I \cdot I)(s)=\int_{0}^{\infty} \ldots \int_{0}^{\infty} \prod_{i=1}^{\mathrm{r}} \mathrm{t}_{\mathrm{i}}^{\mathrm{s}_{\mathrm{i}}-1} \mathrm{I}\left[\mathrm{z}_{1} \mathrm{t}_{1}^{-1}, \ldots \mathrm{z}_{\mathrm{r}} \mathrm{t}_{\mathrm{r}}^{-1}\right] \mathrm{I}^{\prime}\left[\eta_{1} \mathrm{t}_{1}, \ldots, \eta_{\mathrm{r}} \mathrm{t}_{\mathrm{r}}\right] \mathrm{dt}_{1} \ldots \mathrm{dt}_{\mathrm{r}}
\end{aligned}
$$

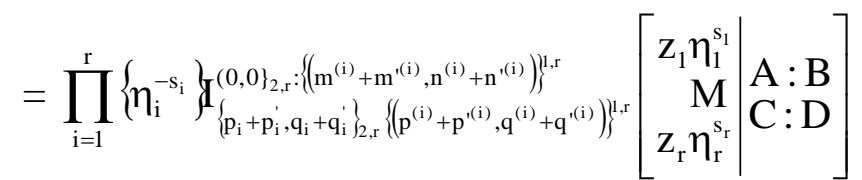

where

$$
\begin{aligned}
& -\min _{1 \leq \mathrm{j} \leq \mathrm{n}^{(\mathrm{i})}} \mathfrak{R}\left(\frac{1-\mathrm{a}_{\mathrm{j}}^{(\mathrm{i})}}{\alpha_{\mathrm{j}}^{(\mathrm{i})}}\right)-\min _{1 \leq \mathrm{j} \leq \mathrm{m}^{(\mathrm{i})}} \mathfrak{R}\left(\frac{\mathrm{b}_{\mathrm{j}}^{\mathrm{i}^{(\mathrm{i})}}}{\beta_{\mathrm{j}}^{\prime^{(\mathrm{i})}}}\right)<\mathfrak{R}\left(\mathrm{s}_{\mathrm{i}}\right)< \\
& \min _{1 \leq \mathrm{j} \leq \mathrm{m}^{(\mathrm{i})}} \mathfrak{R}\left(\frac{\mathrm{b}_{\mathrm{j}}^{(\mathrm{i})}}{\beta_{\mathrm{j}}^{(\mathrm{i})}}\right)+\min _{1 \leq \mathrm{j} \leq \mathrm{n}^{(\mathrm{i})}} \mathfrak{R}\left(\frac{1-\mathrm{a}_{\mathrm{j}}^{(\mathrm{i})}}{\alpha_{\mathrm{j}}^{\mathbf{i}^{(\mathrm{i})}}}\right) \\
& \mathrm{i}=1, \ldots, \mathrm{r} \text { and } \\
& \mathrm{A} \equiv\left\{\left(\mathrm{a}_{\mathrm{ij}} ; \alpha_{\mathrm{ij}}^{(1)}, \ldots, \alpha_{\mathrm{ij}}^{(\mathrm{i})}\right)_{1, \mathrm{p}_{\mathrm{i}}}^{2, \mathrm{r}} ;\left(\mathrm{a}_{\mathrm{ij}}^{\prime}+\sum_{\mathrm{k}=1}^{\prime} \mathrm{s}_{\mathrm{k}} \alpha_{\mathrm{ij}}^{\prime(\mathrm{k})} \alpha_{\mathrm{ij}}^{\prime(1)}, \ldots, \alpha_{\mathrm{ij}}^{\prime(\mathrm{i})}\right)_{1, \mathrm{p}_{\mathrm{i}}^{\prime}}^{2, \mathrm{r}}\right\}, \\
& \mathrm{B} \equiv\left\{\left(a_{j}^{(i)}, \alpha_{j}^{(i)}\right)_{1, p^{(i)}}^{1, r} ;\left(a_{j}^{\prime(i)}+s_{i} \alpha_{j}^{\prime(i)}, \alpha_{j}^{\prime(i)}\right)_{1, p^{(i)}}^{1, r}\right\} \\
& \mathrm{C} \equiv\left\{\left(\mathrm{b}_{\mathrm{ij}} ; \beta_{\mathrm{ij}}^{(1)}, \ldots, \beta_{\mathrm{ij}}^{(\mathrm{i})}\right)_{1, \mathrm{q}_{\mathrm{i}}}^{2, \mathrm{r}} ;\left(\mathrm{b}_{\mathrm{ij}}^{\prime}+\sum_{\mathrm{k}=1}^{\prime} \mathrm{s}_{\mathrm{k}} \beta_{\mathrm{ij}}^{\prime(\mathrm{k})} \beta_{\mathrm{ij}}^{(1)}, \ldots, \beta_{\mathrm{ij}}^{(\mathrm{i})}\right)_{1, \mathrm{q}_{\mathrm{i}}^{\prime}}^{2, \mathrm{r}}\right\} \text {, } \\
& \mathrm{D} \equiv\left\{\left(\mathrm{b}_{\mathrm{j}}^{(\mathrm{i})}, \beta_{\mathrm{j}}^{(\mathrm{i})}\right)_{1, \mathrm{q}^{(\mathrm{i})}}^{1, \mathrm{r}} ;\left(\mathrm{b}_{\mathrm{j}}^{\prime(\mathrm{i})}+\mathrm{s}_{\mathrm{i}} \beta_{\mathrm{j}}^{\prime(\mathrm{i})}, \beta_{\mathrm{j}}^{\prime(\mathrm{i})}\right)_{1, \mathrm{q}^{\prime(\mathrm{i})}}^{1, \mathrm{r}}\right\}
\end{aligned}
$$

\section{The I-Function Transformation Involving R- Variables}

The I-function Transformation of function $\mathrm{f}$ involving $\mathrm{r}$-variables is given as

$$
\hat{\mathrm{f}}\left(\mathrm{y}_{1}, \mathrm{y}_{2}, \ldots \mathrm{y}_{\mathrm{r}}\right)=\mathrm{I}\left[\mathrm{f}\left(\mathrm{x}_{1}, \mathrm{x}_{2}, \ldots \mathrm{x}_{\mathrm{r}}\right) ; \mathrm{y}_{1}, \mathrm{y}_{2}, \ldots \mathrm{y}_{\mathrm{r}}\right]
$$




$$
\begin{gathered}
= \\
\int_{0}^{\infty} \ldots \int_{0}^{\infty} \prod_{\mathrm{i}=1}^{\mathrm{r}} \mathrm{x}_{\mathrm{i}}^{-1} \mathrm{I}\left[\mathrm{y}_{1} \mathrm{x}_{1}, \mathrm{y}_{2} \mathrm{x}_{2}, \ldots \mathrm{y}_{\mathrm{r}} \mathrm{x}_{\mathrm{r}}\right] \mathrm{f}\left(\mathrm{x}_{1}, \ldots ., \mathrm{x}_{\mathrm{r}}\right) \cdot \mathrm{dx}_{1} \ldots \mathrm{dx} \mathrm{x}_{\mathrm{r}} \\
\text { provided that the integral exists. }
\end{gathered}
$$

\section{The Convolution Property}

$$
\left.\begin{array}{ll}
\text { Let } & \hat{\mathrm{k}}(\mathrm{s})=\mathrm{I}\left[\mathrm{k}\left(\mathrm{x}_{1}, \ldots \mathrm{x}_{\mathrm{r}}\right) ; \mathrm{s}_{1}, \ldots, \mathrm{s}_{\mathrm{r}}\right] \\
\text { and } \quad \hat{\mathrm{f}}(\mathrm{s})=\mathrm{I}\left[\mathrm{f}\left(\mathrm{x}_{1}, \ldots \mathrm{x}_{\mathrm{r}}\right) ; \mathrm{s}_{1}, \ldots, \mathrm{s}_{\mathrm{r}}\right]
\end{array}\right\}
$$

Now we obtain an interesting result which expresses the Mellin convolution of I function transforms of two functions of $r$ variables as the I function transform of multiple Mellin convolution of the functions.

$$
\begin{aligned}
& (\hat{\mathrm{k}} * \hat{\mathrm{f}})\left(\mathrm{y}_{1}, \mathrm{y}_{2}, \ldots, \mathrm{y}_{\mathrm{r}}\right)=\int_{0}^{\infty} \ldots \int_{0}^{\infty} \prod_{\mathrm{i}=1}^{\mathrm{r}} \mathrm{z}_{\mathrm{i}}^{-1} \mathrm{I}\left[\mathrm{y}_{1} \mathrm{z}_{1}, \mathrm{y}_{2} \mathrm{z}_{2}, \ldots \mathrm{y}_{\mathrm{r}} \mathrm{z}_{\mathrm{r}}\right] \\
& \quad \cdot\left\{\int_{0}^{\infty} \ldots \int_{0}^{\infty} \prod_{\mathrm{i}=1}^{\mathrm{r}} \mathrm{x}_{\mathrm{i}}^{-1} \mathrm{k}\left(\frac{\mathrm{z}_{1}}{\mathrm{x}_{1}}, \ldots \frac{\mathrm{z}_{\mathrm{r}}}{\mathrm{x}_{\mathrm{r}}}\right) \mathrm{f}\left(\mathrm{x}_{1}, \ldots, \mathrm{x}_{\mathrm{r}}\right) \mathrm{dx_{1 }} \ldots \mathrm{dx} \mathrm{x}_{\mathrm{r}}\right\} \cdot \mathrm{dz_{1 } \ldots d \mathrm { dz } _ { \mathrm { r } }}
\end{aligned}
$$

provided that each side exists.

Proof : The Multiple Mellin transform of $\phi\left(\mathrm{x}_{1} \ldots \mathrm{x}_{\mathrm{r}}\right)$ is given as follows

$$
\mathrm{M}\left\{\phi\left(\mathrm{x}_{1}, \mathrm{x}_{2}, \ldots, \mathrm{x}_{\mathrm{r}}\right) ; \mathrm{s}_{\mathrm{i}}\right\}=\int_{0}^{\infty} \ldots \int_{0}^{\infty} \prod_{\mathrm{i}=1}^{\mathrm{r}} \mathrm{x}_{\mathrm{i}}^{\mathrm{s}_{\mathrm{i}}-1} \phi\left(\mathrm{x}_{1}, \ldots, \mathrm{x}_{\mathrm{r}}\right) \mathrm{dx}_{1} \ldots \mathrm{dx}_{\mathrm{r}}
$$

with the assumptions of absolute convergence of the integrals exist and

$$
\text { let } \quad \mathrm{K}(\mathrm{s})=\mathrm{M}\left\{\mathrm{k}\left(\mathrm{x}_{1} \ldots \mathrm{x}_{\mathrm{r}}\right): \mathrm{s}_{1} \ldots \mathrm{s}_{\mathrm{r}}\right), \mathrm{F}(\mathrm{s})=\mathrm{M}\left\{\mathrm{f}\left(\mathrm{x}_{1} \ldots \mathrm{x}_{\mathrm{r}}\right): \mathrm{s}_{1} \ldots \mathrm{s}_{\mathrm{r}}\right\}
$$

Take Mellin transform of the left hand side of (3.2)

$$
\begin{gathered}
\Delta(\mathrm{s})=\operatorname{M}\left\{(\hat{\mathrm{k}} * \hat{\mathrm{f}}): \mathrm{s}_{1} \ldots \mathrm{s}_{\mathrm{r}}\right\} \\
= \\
\int_{0}^{\infty} \ldots \int_{0}^{\infty} \prod_{\mathrm{i}=1}^{\mathrm{r}} \mathrm{y}_{\mathrm{i}}^{\mathrm{s}_{\mathrm{i}}-1}\left\{\int_{0}^{\infty} \ldots \int_{0}^{\infty} \prod_{\mathrm{i}=1}^{\mathrm{r}} \mathrm{x}_{\mathrm{i}}^{-1} \mathrm{k}\left(\frac{\mathrm{y}_{1}}{\mathrm{x}_{1}} \ldots \frac{\mathrm{y}_{\mathrm{r}}}{\mathrm{x}_{\mathrm{r}}}\right) \mathrm{f}\left(\mathrm{x}_{1}, \ldots, \mathrm{x}_{\mathrm{r}}\right) \mathrm{dx}_{1} \ldots \mathrm{dx} \mathrm{x}_{\mathrm{r}}\right\} \mathrm{dy}_{1} \ldots \mathrm{dy} \mathrm{y}_{\mathrm{r}}
\end{gathered}
$$


On suitable changing the order of two sets of integrations on the right hand side of (3.5) we get

$$
\begin{aligned}
& \Delta(\mathrm{s})=M\left\{(\hat{\mathrm{k}} * \hat{\mathrm{f}}): \mathrm{s}_{1} \ldots \mathrm{s}_{\mathrm{r}}\right\} \\
&= \\
& \int_{0}^{\infty} \ldots \int_{0}^{\infty} \prod_{\mathrm{i}=1}^{\mathrm{r}} \mathrm{x}_{\mathrm{i}}^{-1} \mathrm{f}\left(\mathrm{x}_{1}, \ldots, \mathrm{x}_{\mathrm{r}}\right)\left\{\int_{0}^{\infty} \ldots \int_{0}^{\infty} \prod_{\mathrm{i}=1}^{\mathrm{r}} \mathrm{y}_{\mathrm{i}}^{\mathrm{s}_{\mathrm{i}}-1} \mathrm{k}\left(\frac{\mathrm{y}_{1}}{\mathrm{x}_{1}} \ldots \frac{\mathrm{y}_{\mathrm{r}}}{\mathrm{x}_{\mathrm{r}}}\right) \mathrm{dy}_{1} \ldots \mathrm{dy}_{\mathrm{r}}\right\} \mathrm{dx}_{1} \ldots \mathrm{dx_{ \textrm {r } }} \\
&=\mathrm{M}\left\{\mathrm{f}\left(\mathrm{x}_{1} \ldots \mathrm{x}_{\mathrm{r}}\right): \mathrm{s}_{1} \ldots \mathrm{s}_{\mathrm{r}}\right\} \quad \mathrm{M}\left\{\mathrm{k}\left(\mathrm{y}_{1} \ldots \mathrm{y}_{\mathrm{r}}\right): \mathrm{s}_{1} \ldots \mathrm{s}_{\mathrm{r}}\right\} \\
&=\mathrm{F}(\mathrm{s}) \cdot \mathrm{K}(\mathrm{s})
\end{aligned}
$$

Now using the following result,

$$
\begin{aligned}
& M\left\{\hat{k}\left(y_{1} \ldots y_{r}\right): s_{1} \ldots s_{r}\right\}=M\left\{I\left(y_{1} \ldots y_{r}\right): s_{1} \ldots s_{r}\right\} K(-s) \\
& M\left\{\hat{f}\left(y_{1} \ldots y_{r}\right): s_{1} \ldots s_{r}\right\}=M\left\{I\left(y_{1} \ldots y_{r}\right): s_{1} \ldots s_{r}\right\} F(-s) \text { in (3.7) }
\end{aligned}
$$

which can easily proved as

$$
\begin{aligned}
& \mathrm{M}\left\{\hat{\mathrm{k}}\left(\mathrm{y}_{1} \ldots \mathrm{y}_{\mathrm{r}}\right)\right\}=\int_{0}^{\infty} \ldots \int_{0}^{\infty} \prod_{\mathrm{i}=1}^{\mathrm{r}} \mathrm{y}_{\mathrm{i}}^{\mathrm{s}_{\mathrm{i}}-1}\left\{\int_{0}^{\infty} \ldots \int_{0}^{\infty} \prod_{\mathrm{i}=1}^{\mathrm{r}} \mathrm{x}_{\mathrm{i}}^{-1} \mathrm{I}\left(\mathrm{y}_{1} \mathrm{x}_{1}, \ldots, \mathrm{y}_{\mathrm{r}} \mathrm{x}_{\mathrm{r}}\right) \mathrm{k}\left(\mathrm{x}_{1}, \ldots, \mathrm{x}_{\mathrm{r}}\right) \mathrm{dx}_{1} \ldots \mathrm{dx} \mathrm{x}_{\mathrm{r}}\right\} d \mathrm{dy}_{1} \ldots \mathrm{dy} \mathrm{y}_{\mathrm{r}} \\
& \begin{array}{c}
= \\
\int_{0}^{\infty} \quad \ldots \int_{0}^{\infty} \prod_{\mathrm{i}=1}^{\mathrm{r}} \mathrm{x}_{\mathrm{i}}^{-1} \mathrm{k}\left(\mathrm{x}_{1}, \ldots, \mathrm{x}_{\mathrm{r}}\right) \mathrm{dx}_{1} \ldots \mathrm{dx}_{\mathrm{r}} \int_{0}^{\infty} \ldots \int_{0}^{\infty} \prod_{\mathrm{i}=1}^{\mathrm{r}} \mathrm{y}_{\mathrm{i}}^{\mathrm{s}_{\mathrm{i}}-1} \mathrm{I}\left(\mathrm{y}_{1} \mathrm{x}_{1}, \ldots, \mathrm{y}_{\mathrm{r}} \mathrm{x}_{\mathrm{r}}\right) \mathrm{dy}_{1} \ldots \mathrm{dy_{ \textrm {r } }}
\end{array} \\
& \text { Let } \quad \prod_{\mathrm{i}=1}^{\mathrm{r}} \mathrm{y}_{\mathrm{i}} \mathrm{x}_{\mathrm{i}}=\prod_{\mathrm{i}=1}^{\mathrm{r}} \mathrm{z}_{\mathrm{i}} \\
& \prod_{i=1}^{\mathrm{r}} \mathrm{dy}_{\mathrm{i}}=\frac{\prod_{\mathrm{i}=1}^{\mathrm{r}} \mathrm{dz}_{\mathrm{i}}}{\prod_{\mathrm{i}=1}^{\mathrm{r}} \mathrm{x}_{\mathrm{i}}} \\
& =\int_{0}^{\infty} \ldots \int_{0}^{\infty} \prod_{\mathrm{i}=1}^{\mathrm{r}} \mathrm{x}_{\mathrm{i}}^{-\mathrm{s}_{\mathrm{i}}-1} \mathrm{k}\left(\mathrm{x}_{1}, \ldots, \mathrm{x}_{\mathrm{r}}\right) \mathrm{dx}_{1} \ldots \mathrm{dx} \mathrm{x}_{\mathrm{r}}^{\infty} \ldots \int_{0}^{\infty} \prod_{\mathrm{i}=1}^{\mathrm{r}} \mathrm{z}_{\mathrm{i}}^{\mathrm{s}_{\mathrm{i}}-1} \mathrm{I}\left(\mathrm{z}_{1}, \ldots, \mathrm{z}_{\mathrm{r}}\right) \mathrm{dz}_{1} \ldots \mathrm{dz} \mathrm{z}_{\mathrm{r}} \\
& \mathrm{M}\left\{\hat{\mathrm{k}}\left(\mathrm{y}_{1} \ldots \mathrm{y}_{\mathrm{r}}\right)\right\}=\mathrm{M}\left\{\mathrm{I}\left(\mathrm{y}_{1} \ldots \mathrm{y}_{\mathrm{r}}\right)\right\} \mathrm{K}(-\mathrm{s})
\end{aligned}
$$

Similarly

$$
M\left\{\hat{f}\left(y_{1} \ldots y_{r}\right)\right\}=M\left\{I\left(y_{1} \ldots y_{r}\right)\right\} F(-s)
$$


Hence from (3.7) we get

$$
\Delta(\mathrm{s})=\mathrm{M}\left\{(\hat{\mathrm{k}} * \hat{\mathrm{f}})\left(\mathrm{y}_{1} \ldots \mathrm{y}_{\mathrm{r}}\right)\right\}=M\left\{\hat{\mathrm{k}}\left(\mathrm{y}_{1} \ldots \mathrm{y}_{\mathrm{r}}\right)\right\} \mathrm{M}\left\{\hat{\mathrm{f}}\left(\mathrm{y}_{1} \ldots \mathrm{y}_{\mathrm{r}}\right)\right\}
$$

By using (3.8) \& (3.9) we get

$$
\Delta(\mathrm{s})=\mathrm{M}\left\{\mathrm{I}_{\mathrm{p}, \mathrm{q}: \mathrm{r}}^{\mathrm{m}, \mathrm{n}}\left(\mathrm{y}_{1} \ldots \mathrm{y}_{\mathrm{r}}\right)\right\} \mathrm{K}(-\mathrm{s}) \cdot \mathrm{M}\left\{\mathrm{I}_{\mathrm{p}, \mathrm{q}^{\prime} \mathrm{r}}^{\mathrm{I}^{\prime}, \mathrm{r}^{\prime}}\left(\mathrm{y}_{1} \ldots \mathrm{y}_{\mathrm{r}}\right)\right\} \mathrm{F}(-\mathrm{s})
$$

On the other hand Mellin convolution of the right hand side of (3.2) is

$$
\begin{aligned}
& \Delta^{\prime}(\mathrm{s})=\mathrm{M}\left\{\int_{0}^{\infty} \ldots \int_{0}^{\infty} \prod_{\mathrm{i}=1}^{\mathrm{r}} \mathrm{z}_{\mathrm{i}}^{-1}=\mathrm{I}_{\mathrm{p}+\mathrm{p}^{\prime}, \mathrm{q}+\mathrm{q}^{\prime}}^{\mathrm{m}+\mathrm{m}^{\prime} \mathrm{n} \mathrm{n}^{\prime}}\left[\mathrm{y}_{1} \mathrm{z}_{1}, \ldots, \mathrm{y}_{\mathrm{r}} \mathrm{z}_{\mathrm{r}}\right] \cdot(\mathrm{k} * \mathrm{f})(\mathrm{z}) \mathrm{dz}\right\} \\
& \Delta^{\prime}(\mathrm{s})=\int_{0}^{\infty} \ldots \int_{0}^{\infty} \prod_{\mathrm{i}=1}^{\mathrm{r}} \mathrm{y}_{\mathrm{i}}^{\mathrm{s}_{\mathrm{i}}-1}\left\{\int_{0}^{\infty} \ldots \int_{0}^{\infty} \prod_{\mathrm{i}=1}^{\mathrm{r}} \mathrm{z}_{\mathrm{i}}^{-1} \mathrm{I}\left[\mathrm{y}_{1} \mathrm{z}_{1}, \ldots, \mathrm{y}_{\mathrm{r}} \mathrm{z}_{\mathrm{r}}\right](\mathrm{k} * \mathrm{f})(\mathrm{z}) \mathrm{dz} \mathrm{z}_{1} \ldots d \mathrm{dz}_{\mathrm{r}}\right\} \mathrm{dy}_{1} \ldots d \mathrm{dy}_{\mathrm{r}}
\end{aligned}
$$

Changing the order of two sets of integrations in (3.11), we get

$$
\Delta^{\prime}(\mathrm{s})=\mathrm{M}\left\{\mathrm{I}\left(\mathrm{y}_{1} \mathrm{z}_{1} \ldots \mathrm{y}_{\mathrm{r}} \mathrm{z}_{\mathrm{r}}\right)\right\} \int_{0}^{\infty} \ldots \int_{0}^{\infty} \prod_{\mathrm{i}=1}^{\mathrm{r}} \mathrm{z}_{\mathrm{i}}^{-\mathrm{s}_{\mathrm{i}}-1}(\mathrm{k} * \mathrm{f})(\mathrm{z}) \mathrm{dz} \mathrm{z}_{1} \ldots \mathrm{dz} \mathrm{z}_{\mathrm{r}}
$$

Now applying (1.8) and (3.7)

$$
\left.\Delta^{\prime} \mathrm{s}\right)=\mathrm{M}\left\{\mathrm{I}_{\mathrm{p}, \mathrm{qr}}^{\mathrm{m}, \mathrm{n}}[\xi] ; \mathrm{s}_{1} \ldots \mathrm{s}_{\mathrm{r}}\right\} \cdot \mathrm{M}\left\{\mathrm{I}_{\mathrm{p}^{\prime}, \mathrm{q}_{\mathrm{r}}^{\prime} \mathrm{r}}^{\mathrm{m}^{\prime}, \mathrm{n}^{\prime}}[\eta] ; \mathrm{s}_{1}^{\prime}, \mathrm{s}_{2}^{\prime} \ldots \mathrm{s}_{\mathrm{r}}^{\prime}\right\} \mathrm{K}(-\mathrm{s}) \mathrm{F}(-\mathrm{s})
$$

Equation (3.10) and (3.12) together exhibit the fact that the Mellin transforms of the two sides of (3.2) are equal, and so the two sides of (3.2) must also be equal.

\section{References}

[1] Dutta B.K., Arora L.K., Multiple and Laplace Transforms of I-function of $r$ variables, Journal of Fractinal Calculus and Applications, Vol-1 (2011), 1-8.

[2] Erdelyi A., et al., Table of integral transforms, Vols. I and II, Mc Graw-Hill, New York, London and Toronto, 1954.

[3] Gupta, K.C. and Goyal, S.P., On the two dimensional Mellin convolution, Indian Journal of Mathematics, Vol. 21, No. 3(1979), 177-186.

[4] Gupta, K.C. and Jain, U.C., The H-function II, Proc. Nat. Acad. Sci. India Sect. A 36(1966), 594-609. 
[5] Kumbhat, R.K. and Khan, A.M., Mellin convolution and I-function transformation, Proc. Int. Conf. SSFA, Vol. II, 2001, pp. 93-98.

[6] Prasad T., Singh N.P., Mellin and Laplace transform of the multivariable Ifunction, Ganita Sandesh, 4(2) (1990), 66-70.

[7] Prasad Y.N., Multivariable I-function, Vijanana Parishad Anusandhan Patrika, 29(4) (1986), 231-235.

[8] Prasad Y.N., Yadav G.S., Proc. math. Soc. B.H.U., I (1985), 127-136.

[9] Sharma, C.K. and Shrivastava, S., Some expansion formula for the Ifunction, Nat, Acad, Sci. India, 62 A (1992), 236-239.

[10] Shrivastava, H.M. and Buschman, R.G., Mellin convolution and H-function transformations, Rocky Mountain J. Math. 6(1976), 331-343.

[11] Saxena R.K., Singh Y., On the derivatives of the multivariable I-function, Vijananan Parishad Anusandhan Patrika, 36(2) (1993), 93-38.

[12] Vaishya G.D., Jain R. and Varma R.C., Certain Properties of the I-function, Proc. Nat., Acad. Sci. India. 59(A) II. 1989. 